\title{
Angiotensinogen and Risk of Stroke Events in Patients with Type 2 Diabetes Mellitus
}

\author{
Tao Liu' \\ Weihong Liu $^{2}$ \\ 'Department of Neurology, Tianjin \\ Nankai Hospital, Tianjin, 300100, People's \\ Republic of China; ${ }^{2}$ Department of \\ Traditional Chinese Medicine, Tianjin \\ Fourth Central Hospital, Tianjin, 300I40, \\ People's Republic of China
}

Background: Activation of the renin-angiotensin system (RAS) in diabetic patients is a vital pathophysiological mechanism of cardiovascular complications.

Aim: We aimed to assess whether serum and urinary angiotensinogen levels could predict the risk of stroke events in patients with type 2 diabetes.

Methods: An analysis of the relationships between serum and urinary angiotensinogen levels at baseline and the risk of stroke events was performed in a study consisting of 467 patients with type 2 diabetes with a follow-up of 5 years. Multivariate Cox regression models were built by controlling for a large range of related risk factors.

Results: Kaplan-Meier analysis showed that patients with low estimated glomerular filtration rate (eGFR) $<57 \mathrm{~mL} / \mathrm{min} / 1.73 \mathrm{~m}^{2}$ had a significantly higher risk of stroke events than those with high eGFRs $\left(\geq 57 \mathrm{~mL} / \mathrm{min} / 1.73 \mathrm{~m}^{2}, \mathrm{P}=0.040\right)$. Our results suggested that urinary angiotensinogen levels ( $\mathrm{HR}=2.74,95 \%$ CI $1.50-5.88, P=<0.001)$, but not serum angiotensinogen levels $(\mathrm{HR}=1.42,95 \% \mathrm{CI} 0.95-2.65, P=0.071)$, were independent predictors of the risk of stroke events in patients with type 2 diabetes after adjusting for confounding factors. Similarly, sensitivity analysis also suggested that higher urinary angiotensinogen levels still contributed to an increased risk of stroke events $(\mathrm{HR}=2.71,95 \%$ CI $1.48-5.82, \mathrm{P}<0.001)$ but not serum angiotensinogen levels $(\mathrm{HR}=1.37,95 \% \mathrm{CI} 0.89-2.21, \mathrm{P}=0.104)$. Importantly, we found that significant associations only existed in patients with eGFRs $<60 \mathrm{~mL} / \mathrm{min} / 1.73 \mathrm{~m}^{2}$ $(\mathrm{HR}=2.78,95 \% \mathrm{CI} 1.59-6.30, \mathrm{P}<0.001)$ but not in patients with eGFRs $\geq 60 \mathrm{~mL} / \mathrm{min} / 1.73 \mathrm{~m}^{2}$ ( $\mathrm{HR}=1.39,95 \%$ CI $0.95-3.53, \mathrm{P}=0.054)$.

Conclusion: The study suggested that elevated urinary angiotensinogen levels were correlated with a higher risk of stroke events in patients with type 2 diabetes mellitus.

Keywords: angiotensinogen diabetes mellitus, stroke, Cox proportional hazard regression

\section{Introduction}

Type 2 diabetes has been considered a vital risk factor for promoting the occurrence and/or development of cardiovascular disease (CVD), such as stroke and coronary heart disease (CHD), and CVD mortality. ${ }^{1-3}$ Previous studies have shown that individuals with type 2 diabetes may have different severities of the disease, which depends on the presence of comorbidities or other risk factors. ${ }^{4}$ Wellunderstood risk heterogeneity and identifying individuals at long-term risk could help improve and personalize cardiovascular risk management for these individuals with type 2 diabetes.

Activation of the renin-angiotensin system (RAS) is a vital pathophysiological mechanism of CVD and renal insufficiency in diabetic patients. ${ }^{5}$ Many previous observational studies have demonstrated that inhibiting the RAS, which is
Department of Neurology, Tianjin Nankai Hospital, NO. 6, Changjiang Road, Nankai

District, Tianjin, 300I00, People's

Republic of China

Email taoliu|23123@I26.com 
currently the front-line treatment for diabetic nephropathy, ${ }^{5}$ could delay worsening renal function and reduce the risk of CVD morbidity and mortality in patients with diabetes. ${ }^{6-8}$ Importantly, although RAS inhibition has shown many beneficial effects, not all patients showed significant improvements in the prognosis of CVD complications. Hence, accurately estimating the active state of the intrarenal RAS might provide a good opportunity to help identify whether diabetic patients are at high risk of poor prognosis. The kidney has all parts of the RAS pathway that can produce angiotensinogen, which further promotes the production of angiotensin II (angII). ${ }^{9}$ AngII, produced by the kidney, has been reported to pose a key role in renal function and hemodynamics, affecting the development of cardiovascular pathology. $^{10}$

Recently, several cross-sectional studies have reported that urinary angiotensinogen may be considered a potential biomarker of renal dysfunction in hypertensive patients. $^{11,12}$ However, whether urinary and/or serum levels of angiotensinogen can be considered potential biomarkers for predicting stroke risk is still unclear. In this study, we measured urinary and serum levels of angiotensinogen in patients with type 2 diabetes. We aimed to assess whether angiotensinogen levels are associated with stroke prognosis.

\section{Methods}

\section{Study Sample}

We studied 488 hospitalized patients with type 2 diabetes from Tianjin Nankai Hospital in China between January 2009 and December 2015. None of the included patients had other serious chronic diseases, such as cancers, liver diseases, or respiratory diseases, before admission. After discharge, the patients with type 2 disease were contacted by telephone. A total of 21 patients with type 2 diabetes were excluded from this cohort study due to the diagnosis of serious chronic diseases within three months before admission, including neoplastic diseases $(\mathrm{N}=10)$, liver diseases $(\mathrm{N}=7)$ and other serious diseases $(\mathrm{N}=4)$. The diagnostic criteria for type 2 diabetes were determined by 3 endocrinologists. ${ }^{13}$ For the purposes of this study, during a mean follow-up of 5 years, ischemic or hemorrhagic stroke requiring hospitalization was defined as an endpoint event. The endpoint event was diagnosed by two neurologists. The Ethics Committee of Tianjin Nankai Hospital approved this study. This was a retrospective study, so this study applied for patients informed exemption according to the Declaration of Helsinki guidelines.

\section{Follow-Up}

The included diabetic patients were followed up by telephone and/or reviewing, until the occurrence of endpoint events. The endpoint events of this study were defined as ischemic stroke requiring rehospitalization, hemorrhagic stroke requiring rehospitalization and death caused by stroke. During the 5-year follow-up period, 7 patients with diabetes were lost to follow-up.

\section{Measurement of Serum and Urinary Angiotensinogen Levels}

Fasting venous blood samples were obtained from the diabetic patients in the first morning after inclusion. The concentrations of angiotensinogen in urine and serum samples were measured by using enzyme-linked immunosorbent assays (ELISA) at baseline. ${ }^{14}$ Angiotensinogen concentrations were tested three times in each patient, and the average value of the three results was used for statistical analysis. The interassay and intra-assay coefficients of variability for the serum and urine angiotensinogen assays were $6.5 \%$ and $4.5 \%$, respectively.

The blood samples were also measured for serum albumin (ABL), glycosylated hemoglobin (HbA1c), hemoglobin (Hb), low density lipoprotein (LDL), high sensitivity C-reactive protein (hs-CRP) and high density lipoprotein (HDL) levels and were tested at the same time by using immunoassay on an ELECSYS2010 instrument (Roche Diagnostics, Germany). Serum levels of the estimated glomerular filtration rate (eGFR) were calculated by using the Chronic Kidney Disease (CKD) Epidemiology Collaboration equation. ${ }^{15}$ For research purposes, an eGFR $<60 \mathrm{~mL} / \mathrm{min} / 1.73 \mathrm{~m}^{2}$ was considered renal insufficiency.

\section{Statistical Analysis}

All of the data were analyzed by using SPSS 22.0, and a $P \leq$ 0.05 was considered to be statistically significant. The Kolmogorov-Smirnov test was used to analyze the normality of the data. $t$-tests or chi-square tests were performed to compare the two groups $\left(\mathrm{eGFR} \geq 60 \mathrm{~mL} / \mathrm{min} / 1.73 \mathrm{~m}^{2}\right.$ and $\mathrm{eGFR}<60 \mathrm{~mL} / \mathrm{min} / 1.73 \mathrm{~m}^{2}$ ). In the multivariate analysis, Cox regression analysis was performed to identify the independent values of serum and urinary angiotensinogen levels at baseline on predicting the risk of stroke events in patients with 
type 2 diabetes. To further evaluate the independent association, we further excluded the effect of "duration of diabetes" by sensitivity analysis. Moreover, we also analyzed the association between serum and urinary angiotensinogen levels at baseline and the risk of stroke events during the follow-up period using stratified analysis by adding "taking RAS inhibitors" and "an eGFR $\geq 60 \mathrm{~mL} / \mathrm{min} / 1.73 \mathrm{~m}$ ". . Additionally, an endpoint (stroke event)-free curve was constructed by the Kaplan-Meier method, and the Log rank test was performed.

\section{Results}

\section{Clinical Characteristics of the 467 Patients with Type 2 Diabetes at Baseline} The clinical characteristics of the patients with type 2 diabetes at baseline are presented in Table 1. According to the median value of the eGFR $\left(57 \mathrm{~mL} / \mathrm{min} / 1.73 \mathrm{~m}^{2}\right)$, all the patients were divided into two groups. The patients with low eGFRs $\left(<57 \mathrm{~mL} / \mathrm{min} / 1.73 \mathrm{~m}^{2}\right)$ tended to have longer durations of diabetes, higher systolic and diastolic blood pressures, and higher rates of ever being a smoker, ever being a drinker, taking RAS inhibitors and having a CVD history, compared with patients with high eGFRs $\left(\geq 57 \mathrm{~mL} / \mathrm{min} / 1.73 \mathrm{~m}^{2}\right.$, all $\mathrm{P}<0.05$ ). For laboratory measurements, the patients with low eGFRs had higher levels of urinary angiotensinogen, LDL, HbAlc and Hs-CRP and lower levels of ALB and $\mathrm{Hb}$ than those with high eGFRs (all $\mathrm{P}<0.05$ ). Interestingly, serum angiotensinogen was not significantly different between the two groups $(\mathrm{P}>0.05)$.

Cox Proportional Hazard Analysis for the Associations Between Serum and Urinary Angiotensinogen Levels and Stroke Events in Patients with Type 2 Diabetes

All included patients were prospectively followed up for a median period of 5 years, and 47 patients had stroke events (including ischemic and hemorrhagic stroke). Kaplan-Meier

Table I Clinical Characteristics in 467 Patients with Type 2 Diabetes at Baseline

\begin{tabular}{|c|c|c|c|}
\hline Variables & $\begin{array}{c}\text { eGFR } \geq 57 \mathrm{~mL} / \mathrm{min} / 1.73 \mathrm{~m}^{2} \\
(\mathrm{n}=233)\end{array}$ & $\begin{array}{c}\text { eGFR<57 } \mathrm{mL} / \mathrm{min} / 1.73 \mathrm{~m}^{2} \\
(\mathrm{n}=234)\end{array}$ & P value \\
\hline Age (years) & $61.8 \pm 7.5$ & $62.4 \pm 7.6$ & 0.144 \\
\hline Gender (male), n (\%) & $110(47.2)$ & $123(52.6)$ & 0.219 \\
\hline BMI $\left(\mathrm{kg} / \mathrm{m}^{2}\right)$ & $22.5 \pm 6.1$ & $23.1 \pm 7.0$ & 0.102 \\
\hline Ever smoker, n (\%) & $101(43.3)$ & $112(47.9)$ & 0.048 \\
\hline Ever drinker, n (\%) & $107(45.5)$ & $125(53.4)$ & 0.042 \\
\hline Duration of diabetes (years) & $10 \pm 6$ & $15 \pm 8$ & $<0.001$ \\
\hline Systolic blood pressure (mmHg) & $137 \pm 14$ & $147 \pm 18$ & $<0.001$ \\
\hline Diastolic blood pressure $(\mathrm{mmHg})$ & $79 \pm 8$ & $83 \pm 10$ & $<0.001$ \\
\hline Taking RAS inhibitors, n (\%) & $35(15.0)$ & $87(37.2)$ & $<0.001$ \\
\hline Stroke events during follow-up, $\mathrm{n}$ (\%) & $15(6.4)$ & $32(13.7)$ & 0.011 \\
\hline \multicolumn{4}{|l|}{ CVD history } \\
\hline Hypertension, n (\%) & $67(28.8)$ & $95(40.6)$ & 0.010 \\
\hline Coronary heart disease, $\mathrm{n}(\%)$ & II (4.7) & $34(14.5)$ & $<0.001$ \\
\hline Heart failure, $\mathrm{n}(\%)$ & $8(3.4)$ & $17(7.3)$ & 0.047 \\
\hline Others, n (\%) & $4(1.7)$ & $6(2.5)$ & 0.098 \\
\hline \multicolumn{4}{|l|}{ Laboratory measurements } \\
\hline Log-transformed values of angiotensinogen & $23.5 \pm 4.1$ & $23.6 \pm 4.4$ & 0.719 \\
\hline $\begin{array}{l}\text { Log-transformed values of urinary } \\
\text { angiotensinogen }\end{array}$ & $1.3 \pm 0.4$ & $2.1 \pm 0.7$ & $<0.001$ \\
\hline HDL (mmol/L) & $1.3 \pm 0.3$ & $1.2 \pm 0.2$ & 0.059 \\
\hline LDL (mmol/L) & $2.2 \pm 1.1$ & $2.7 \pm 1.3$ & $<0.001$ \\
\hline ALB $(g / L)$ & $37.4 \pm 6.2$ & $34.2 \pm 4.6$ & $<0.001$ \\
\hline $\mathrm{Hb}(\mathrm{g} / \mathrm{L})$ & $122 \pm 10$ & $113 \pm 8$ & 0.008 \\
\hline $\mathrm{HbAlc}(\%)$ & $7.2 \pm 0.4$ & $7.9 \pm 0.5$ & $<0.001$ \\
\hline $\mathrm{Hs}-\mathrm{CRP}(\mathrm{pg} / \mathrm{mL})$ & $7.1(3.4-10.2)$ & $9.4(4.9-14.7)$ & $<0.001$ \\
\hline
\end{tabular}

Abbreviations: eGFR, estimated glomerular filtration rate; BMI, body mass index; RAS, renin-angiotensin system; CVD, cardiovascular disease; HDL, high density lipoprotein; LDL, low density lipoprotein; ALB, albumin; Hb, hemoglobin; HbAlc, glycosylated hemoglobin; hs-CRP, hs-Creactive protein. 


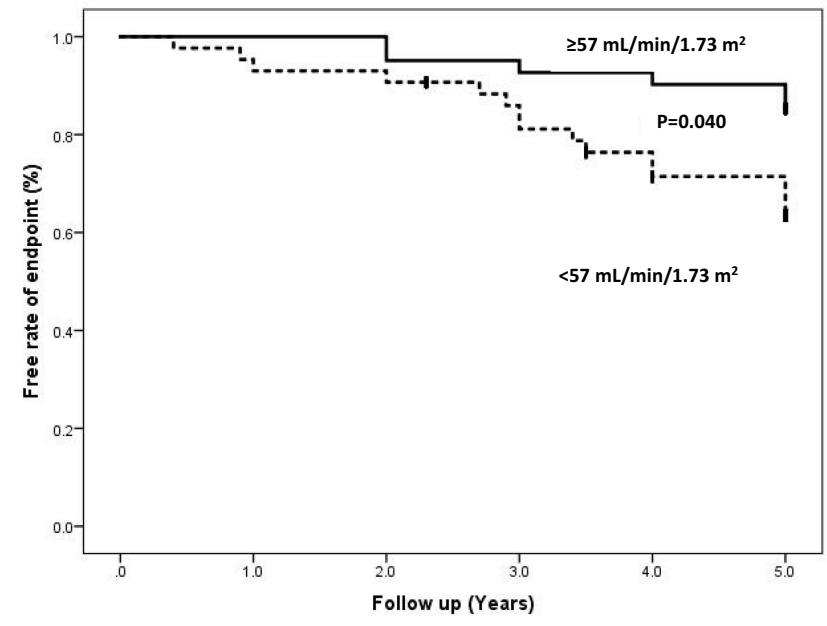

Figure I Kaplan-Meier analysis of the endpoint-free curve stratified into 2 groups by median level of the eGFR.

analysis showed that patients with low eGFRs $(<57 \mathrm{~mL} / \mathrm{min} /$ $1.73 \mathrm{~m}^{2}$ ) had a significantly higher rate of stroke events than those with high eGFRs (Figure 1, $\mathrm{P}=0.040$ ). To further investigate the potential risk for stroke events, a multivariate Cox proportional hazard regression model was used. Urinary angiotensinogen levels $(\mathrm{HR}=2.78,95 \%$ CI 1.54-5.94, $P=<0.001)$ were associated with an increased risk of stroke events when adjustments for age, sex, BMI, ever smoking and ever drinking were made, which was similar to serum angiotensinogen levels $(\mathrm{HR}=1.54,95 \% \mathrm{CI}$ 1.10-3.27, $P=0.037$ ) in Model 1 (Table 2). The significant associations changed slightly after adding systolic and diastolic blood pressures and CVD history in Model 1. After continuing to add the laboratory measurements into Model 2 , our results suggested that urinary angiotensinogen levels $(\mathrm{HR}=2.74,95 \%$ CI 1.50-5.88, $P=<0.001$, Model 3) were an independent predictor for the risk of stroke events in patients with type 2 diabetes, but not serum angiotensinogen levels (HR=1.42, 95\% CI 0.95-2.65, $P=0.071$, Model 3).

We performed an additional sensitivity analysis to evaluate the associations of urinary and serum angiotensinogen levels with the risk of stroke events in patients with type 2 diabetes by adding "duration of diabetes" as a covariate (Table 3 ). Similarly, the results also suggested that higher urinary angiotensinogen levels still contributed to an increased risk of stroke events $(\mathrm{HR}=2.71,95 \%$ CI 1.48-5.82, $\mathrm{P}<0.001$, Model 3), but not serum angiotensinogen levels $(\mathrm{HR}=1.37$, 95\% CI 0.89-2.21, $\mathrm{P}=0.104$, Model 3), after adjusting for the confounding factors.

Table 2 Cox Proportional Hazard Analysis for the Association Between Urinary and Serum Angiotensinogen Levels and Stroke Events in Patients with Type 2 Diabetes

\begin{tabular}{|c|c|c|c|}
\hline Variables & Model I & Model 2 & Model 3 \\
\hline Log-transformed values of angiotensinogen, increased I SD & $1.54(1.10-3.27)$ & $1.50(1.04-3.02)$ & $1.42(0.95-2.65)$ \\
\hline$P$ value & 0.037 & 0.049 & 0.071 \\
\hline Log-transformed values of urinary angiotensinogen, increased I SD & $2.78(1.54-5.94)$ & $2.75(1.51-5.89)$ & $2.74(1.50-5.88)$ \\
\hline$P$ value & $<0.001$ & $<0.001$ & $<0.001$ \\
\hline
\end{tabular}

Notes: Model I: adjusted for age, gender, BMI, ever smoker and ever drinker. Model 2: adjusted for age, gender, BMI, ever smoker, ever drinker, systolic and diastolic blood pressure and CVD history. Model 3: adjusted for age, gender, BMI, ever smoker, ever drinker, systolic and diastolic blood pressure, CVD history and laboratory measurements.

Abbreviations: eGFR, estimated glomerular filtration rate; BMI, body mass index; SD, standard deviation.

Table 3 Sensitivity Analysis for the Association Between Urinary and Serum Angiotensinogen Levels and Stroke Events in Patients with Type 2 Diabetes

\begin{tabular}{|l|c|c|c|}
\hline Variables & Model I & Model 2 & Model 3 \\
\hline Log-transformed values of angiotensinogen, increased I SD & $1.43(I .09-3.16)$ & $1.40(0.94-2.7 I)$ & $1.37(0.89-2.2 I)$ \\
P value & 0.047 & 0.069 & 0.104 \\
Log-transformed values of urinary angiotensinogen, increased I SD & $2.74(1.52-5.90)$ & $2.72(1.50-5.84)$ & $2.7 I(1.48-5.82)$ \\
P value & $<0.001$ & $<0.001$ & $<0.001$ \\
\hline
\end{tabular}

Notes: Model I: adjusted for age, gender, BMI, ever smoker, ever drinker and duration of diabetes. Model 2: adjusted for age, gender, BMI, ever smoker, ever drinker, systolic and diastolic blood pressure, CVD history and duration of diabetes. Model 3: adjusted for age, gender, BMI, ever smoker, ever drinker, systolic and diastolic blood pressure, CVD history, laboratory measurements and duration of diabetes.

Abbreviations: eGFR, estimated glomerular filtration rate; BMI, body mass index; SD, standard deviation. 
Table 4 Stratified Analysis for the Association Between Urinary and Serum Angiotensinogen Levels and Stroke Events in Patients with Type 2 Diabetes by "Taking RAS Inhibitors"

\begin{tabular}{|c|c|c|c|}
\hline Variables & Model I & Model 2 & Model 3 \\
\hline \multicolumn{4}{|l|}{ With taking RAS inhibitors } \\
\hline Log-transformed values of angiotensinogen, increased I SD & $1.42(1.08-3.14)$ & $1.38(0.92-2.68)$ & $1.35(0.86-2.20)$ \\
\hline$P$ value & 0.049 & 0.071 & 0.132 \\
\hline Log-transformed values of urinary angiotensinogen, increased I SD & $2.70(1.51-5.84)$ & $2.67(1.47-5.80)$ & $2.64(1.45-5.78)$ \\
\hline$P$ value & $<0.001$ & $<0.001$ & $<0.001$ \\
\hline \multicolumn{4}{|l|}{ Without taking RAS inhibitors } \\
\hline Log-transformed values of angiotensinogen, increased I SD & $1.44(1.09-3.19)$ & $\mathrm{I} .43(0.96-2.77)$ & $1.37(0.93-2.25)$ \\
\hline$P$ value & 0.046 & 0.065 & 0.100 \\
\hline Log-transformed values of urinary angiotensinogen, increased I SD & $2.77(1.57-6.12)$ & $2.76(1.58-6.05)$ & $2.74(1.60-6.01)$ \\
\hline$P$ value & $<0.001$ & $<0.001$ & $<0.001$ \\
\hline
\end{tabular}

Notes: Model I: adjusted for age, gender, BMI, ever smoker, ever drinker and duration of diabetes. Model 2: adjusted for age, gender, BMI, ever smoker, ever drinker, systolic and diastolic blood pressure, CVD history and duration of diabetes. Model 3: adjusted for age, gender, BMI, ever smoker, ever drinker, systolic and diastolic blood pressure, CVD history, laboratory measurements and duration of diabetes.

Abbreviations: eGFR, estimated glomerular filtration rate; BMI, body mass index; SD, standard deviation.

Table 5 Stratified Analysis for the Association Between Urinary and Serum Angiotensinogen Levels and Stroke Events in Patients with Type 2 Diabetes by "Egfr $\geq 60 \mathrm{~mL} / \mathrm{Min} / \mathrm{I} .73 \mathrm{M}^{2}$ ",

\begin{tabular}{|c|c|c|c|}
\hline Variables & Model I & Model 2 & Model 3 \\
\hline \multicolumn{4}{|l|}{ eGFR $\geq 60 \mathrm{~mL} / \mathrm{min} / 1.73 \mathrm{~m}^{2}$} \\
\hline Log-transformed values of angiotensinogen, increased I SD & $1.43(1.07-3.12)$ & $1.40(0.94-2.72)$ & $1.38(0.90-2.31)$ \\
\hline$P$ value & 0.049 & 0.070 & 0.129 \\
\hline Log-transformed values of urinary angiotensinogen, increased I SD & $1.51(1.11-4.21)$ & $1.46(1.01-3.82)$ & $1.39(0.95-3.53)$ \\
\hline$P$ value & 0.034 & 0.045 & 0.054 \\
\hline \multicolumn{4}{|l|}{ eGFR<60 mL/min/l.73 $\mathrm{m}^{2}$} \\
\hline Log-transformed values of angiotensinogen, increased I SD & $1.47(1.11-3.34)$ & $1.46(0.97-2.89)$ & $1.43(0.99-2.36)$ \\
\hline$P$ value & 0.042 & 0.063 & 0.097 \\
\hline Log-transformed values of urinary angiotensinogen, increased I SD & $2.82(1.63-6.43)$ & $2.80(1.61-6.39)$ & $2.78(1.59-6.30)$ \\
\hline$P$ value & $<0.001$ & $<0.001$ & $<0.001$ \\
\hline
\end{tabular}

Notes: Model I: adjusted for age, gender, BMI, ever smoker, ever drinker and duration of diabetes. Model 2: adjusted for age, gender, BMI, ever smoker, ever drinker, systolic and diastolic blood pressure, CVD history and duration of diabetes. Model 3: adjusted for age, gender, BMI, ever smoker, ever drinker, systolic and diastolic blood pressure, CVD history, laboratory measurements and duration of diabetes.

Abbreviations: eGFR, estimated glomerular filtration rate; BMI, body mass index; SD, standard deviation.

\section{Stratified Analysis of the Associations \\ Between Urinary and Serum}

Angiotensinogen Levels and Stroke Events in Patients with Type 2 Diabetes by "Taking RAS Inhibitors" and "an eGFR $\geq 60 \mathrm{~mL} / \mathrm{Min} /$ I.73 $\mathrm{M}^{2}$ "

Stratified analysis was performed by adding "taking RAS inhibitors" and "an eGFR $\geq 60 \mathrm{~mL} / \mathrm{min} / 1.73 \mathrm{~m}$ " as covariates. Our results still showed that the association between urinary angiotensinogen levels and the risk of stroke events in patients with type 2 diabetes was significant $(\mathrm{HR}=2.64,95 \% \mathrm{CI} 1.45$ 5.78, $\mathrm{P}<0.001$, Model 3) and was not affected by "taking RAS inhibitors", as shown in Table 4. Importantly, the significant association was affected by "an eGFR $\geq 60 \mathrm{~mL} / \mathrm{min} / 1.73 \mathrm{~m}$ ", as shown in Table 5 . We found that a significant association existed only in patients with eGFRs $<60 \mathrm{~mL} / \mathrm{min} / 1.73 \mathrm{~m}^{2}$ (HR $=2.78,95 \%$ CI 1.59-6.30, $\mathrm{P}<0.001$, Model 3$)$ and not in patients with eGFRs $\geq 60 \mathrm{~mL} / \mathrm{min} / 1.73 \mathrm{~m}^{2}(\mathrm{HR}=1.39,95 \% \mathrm{CI}$ 0.95-3.53, $\mathrm{P}=0.054$, Model 3). In addition, serum angiotensinogen levels still had no association with the risk of stroke events in the stratified analysis.

\section{Discussion}

In the present study, our baseline data suggested that patients with higher urinary levels of angiotensinogen had lower eGFRs. However, serum levels of angiotensinogen were not associated with the eGFR. Moreover, Cox regression analysis suggested that diabetic patients with high levels of urinary angiotensinogen had a high rate of 
stroke events. Our results documented that increasing urinary angiotensinogen levels were associated with a higher risk for stroke events in diabetic patients. Furthermore, the significant relationship of urinary angiotensinogen levels with stroke risk can be affected by renal function.

Although previous studies have documented that angiotensinogen can be produced and secreted from both the liver and kidneys, ${ }^{10}$ serum and urinary levels of angiotensinogen originating from different sources pose different impacts on renal function. ${ }^{16,17}$ Existing evidence suggests that human angiotensinogen cannot be detected in urine obtained from hypertensive and nonhypertensive rats that were injected with human angiotensinogen, which may be explained by the limited glomerular permeability of circulating angiotensinogen and/or degrading angiotensinogen in tubules. ${ }^{18}$ Under normal renal structure and function, it has been reported that angiotensinogen is expressed in proximal tubular cells and released into the ureter. ${ }^{10}$ However, in the case of hyperglycemia, the expression of angiotensinogen is significantly increased in proximal tubular cells. ${ }^{19,20}$ Furthermore, some clinical investigations have reported that diabetic patients have higher urinary angiotensinogen levels, ${ }^{21}$ whereas there was no difference in serum angiotensinogen levels between diabetic patients and control individuals. ${ }^{21}$ This previous evidence may suggest that blood angiotensinogen is not a direct source of urinary angiotensinogen. Consistently, our results also suggested that urinary angiotensinogen originates locally from the kidney instead of serum.

Increased angiotensinogen expression in tubules can promote the activation of the intrarenal RAS. Consistent with our study, diabetic patients with low eGFRs had a greater increase in urinary angiotensinogen levels than serum angiotensinogen levels. One similar finding showed that CKD patients with low eGFRs documented higher urinary angiotensinogen levels, suggesting a negative relationship between urinary angiotensinogen levels and renal function. ${ }^{22}$ Studies have long confirmed the correlation between abnormal renal function and CVDs. ${ }^{23}$ Our multivariate correlational analysis reported that elevated levels of urinary angiotensinogen contributed to high stroke risk. Hence, the increased levels of urinary angiotensinogen might contribute to the pathological development of stroke, which may be explained by renal dysfunction and/or the incidence of CVD caused by diabetes mellitus.

Our results have several obvious strengths. On the one hand, in the first morning after admission, blood and urinary tests can better reflect the levels of serum and urinary angiotensinogen in diabetic patients. We are the first to find that urinary angiotensinogen can be considered a valuable predictor for endpoints (stroke events requiring rehospitalization) in diabetic patients. On the other hand, our study confirmed complete follow-up and standardized adjudication of the endpoint, so our results are very reliable. Certainly, this study also has several limitations, including a small sample size in a single center. First, although many various confounding factors, including renal function, were adjusted in our study, which may be the most important factor influencing urinary angiotensinogen levels, some other potential confounding factors were not eliminated due to other unknown determinants of urinary angiotensinogen levels. Second, because urinary levels of angiotensinogen were measured in the first morning after admission, the time-dependent variables after discharge were not assessed, which might cause survivorship biases. Third, using many covariates in our Cox regression analysis may have caused overfitting of the model, leading to bias in the results. Finally, we did not further investigate the mechanisms underlying the association of urinary angiotensinogen levels with stroke. Additionally, our study only included the Asian race and limits the generalizability of our results to other races, such as white and black races. In summary, these limitations should be considered in future studies to elaborate on this work.

\section{Conclusions}

Our results suggested that elevated urinary levels of angiotensinogen contributed to higher stroke risk in diabetic patients. Reducing urinary levels of angiotensinogen might be a new biomarker to reduce stroke risk.

\section{Funding}

There is no funding to report.

\section{Disclosure}

The authors report no conflicts of interest in this work.

\section{References}

1. Roger VL, Go AS, Lloyd-Jones DM, et al; American Heart Association Statistics Committee and Stroke Statistics Subcommittee. Heart disease and stroke statistics-2011 update: a report from the American Heart Association. Circulation. 2011;123(4):e18-e209. doi:10.1161/CIR.0b013e3182009701

2. Selvin E, Juraschek SP, Coresh J. Kidney disease in people with diabetes: the expanding epidemic. Am J Kidney Dis. 2012;59 (3):340-342. doi:10.1053/j.ajkd.2011.11.016

3. Foster MC, Rawlings AM, Marrett E, et al. Cardiovascular risk factor burden, treatment, and control among adults with chronic kidney disease in the United States. Am Heart J. 2013;166(1):150-156. doi:10.1016/j.ahj.2013.03.016

4. Di Angelantonio E, Kaptoge S, Wormser D, et al.; Emerging Risk Factors Collaboration. Association of cardiometabolic multimorbidity with mortality. JAMA. 2015;314(1):52-60. doi:10.1001/ jama.2015.7008 
5. Koya D, Araki S, Haneda M. Therapeutic management of diabetic kidney disease. J Diabetes Investig. 2011;2(4):248-254. doi:10.1111/ j.2040-1124.2011.00112.x

6. Lewis EJ, Hunsicker LG, Clarke WR, et al.; Collaborative Study Group. Renoprotective effect of the angiotensin-receptor antagonist irbesartan in patients with nephropathy due to type 2 diabetes. $N$ Engl $J$ Med. 2001;345(12):851-860. doi:10.1056/NEJMoa011303

7. Brenner BM, Cooper ME, de Zeeuw D, et al.; RENAAL Study Investigators. Effects of losartan on renal and cardiovascular outcomes in patients with type 2 diabetes and nephropathy. $N$ Engl $J$ Med. 2001;345(12):861-869. doi:10.1056/NEJMoa011161

8. Fried LF, Emanuele N, Zhang JH, et al.; VA NEPHRON-D Investigators. Combined angiotensin inhibition for the treatment of diabetic nephropathy. $N$ Engl J Med. 2013;369(20):1892-1903 doi:10.1056/NEJMoa1303154

9. Ingelfinger JR, Zuo WM, Fon EA, Ellison KE, Dzau VJ. In situ hybridization evidence for angiotensinogen messenger RNA in the rat proximal tubule. An hypothesis for the intrarenal renin angiotensin system. J Clin Invest. 1990;85(2):417-423. doi:10.1172/ JCI114454

10. Kobori H, Nangaku M, Navar LG, Nishiyama A. The intrarenal renin-angiotensin system: from physiology to the pathobiology of hypertension and kidney disease. Pharmacol Rev. 2007;59 (3):251-287. doi:10.1124/pr.59.3.3

11. Kobori H, Alper AB Jr, Shenava R, et al. Urinary angiotensinogen as a novel biomarker of the intrarenal renin-angiotensin system status in hypertensive patients. Hypertension. 2009;53(2):344-350. doi:10.1161/HYPERTENSIONAHA.108.123802

12. Kobori H, Ohashi N, Katsurada A, et al. Urinary angiotensinogen as a potential biomarker of severity of chronic kidney diseases. $\mathrm{J} \mathrm{Am}$ Soc Hypertens. 2008;2(5):349-354. doi:10.1016/j.jash.2008.04.008

13. Chatterjee S, Khunti K, Davies MJ. Type 2 diabetes. Lancet. 2017;389(10085):2239-2251. doi:10.1016/S0140-6736(17)30058-2

14. Katsurada A, Hagiwara Y, Miyashita K, et al. Novel sandwich ELISA for human angiotensinogen. Am J Physiol Renal Physiol. 2007;293 (3):F956-60. doi:10.1152/ajprenal.00090.2007

15. Krogh J, Benros ME, Martin Balslev J, et al. The association between depressive symptoms, cognitive function, and inflammation in major depression[J]. Brain Behav Immun. 2014;35(1):70-76. doi:10.1016/j. bbi.2013.08.014
16. Sawaguchi M, Araki SI, Kobori H, et al. Association between urinary angiotensinogen levels and renal and cardiovascular prognoses in patients with type 2 diabetes mellitus. J Diabetes Investig. 2012;3 (3):318-324. doi:10.1111/j.2040-1124.2011.00172.x

17. Yasue S, Masuzaki H, Okada S, et al. Adipose tissue-specific regulation of angiotensinogen in obese humans and mice: impact of nutritional status and adipocyte hypertrophy. Am J Hypertens. 2010;23 (4):425-431. doi:10.1038/ajh.2009.263

18. Kobori H, Nishiyama A, Harrison-Bernard LM, Navar LG. Urinary angiotensinogen as an indicator of intrarenal Angiotensin status in hypertension. Hypertension. 2003;41(1):42-49. doi:10.1161/01. HYP.0000050102.90932.CF

19. Ohashi N, Urushihara M, Satou R, Kobori H. Glomerular angiotensinogen is induced in mesangial cells in diabetic rats via reactive oxygen species-ERK/JNK pathways. Hypertens Res. 2010;33 (11):1174-1181. doi:10.1038/hr.2010.143

20. Hsieh TJ, Zhang SL, Filep JG, Tang SS, Ingelfinger JR, Chan JS High glucose stimulates angiotensinogen gene expression via reactive oxygen species generation in rat kidney proximal tubular cells. Endocrinology. 2002;143(8):2975-2985. doi:10.1210/endo.1 43.8.8931

21. Saito T, Urushihara M, Kotani Y, Kagami S, Kobori H. Increased urinary angiotensinogen is precedent to increased urinary albumin in patients with type 1 diabetes. Am J Med Sci. 2009;338(6):478-480. doi:10.1097/MAJ.0b013e3181b90c25

22. Yamamoto T, Nakagawa T, Suzuki H, et al. Urinary angiotensinogen as a marker of intrarenal angiotensin II activity associated with deterioration of renal function in patients with chronic kidney disease. J Am Soc Nephrol. 2007;18(5):1558-1565. doi:10.1681/ ASN.2006060554

23. Annon CP, Perkovic V, Agarwal R, et al. Evaluating the Effects of Canagliflozin on Cardiovascular and Renal Events in Patients With Type 2 Diabetes Mellitus and Chronic Kidney Disease According to Baseline HbA1c, Including Those With HbA1c $<7 \%$ : results from the credence trial. Circulation. 2020;141(5):407-410. doi:10.1161/ CIRCULATIONAHA.119.044359

Diabetes, Metabolic Syndrome and Obesity: Targets and Therapy

Dovepress

\section{Publish your work in this journal}

Diabetes, Metabolic Syndrome and Obesity: Targets and Therapy is an international, peer-reviewed open-access journal committed to the rapid publication of the latest laboratory and clinical findings in the fields of diabetes, metabolic syndrome and obesity research. Original research, review, case reports, hypothesis formation, expert opinion and commentaries are all considered for publication. The manuscript management system is completely online and includes a very quick and fair peer-review system, which is all easy to use. Visit http://www.dovepress.com/testimonials.php to read real quotes from published authors. 